\title{
NUMERICAL SIMULATION OF SLUMP FLOW TEST OF CEMENT PASTE COMPOSITES
}

\author{
JIŘÍ NĚMEČEK*, JIŘí NĚMEČEK \\ Czech Technical University in Prague, Faculty of Civil Engineering, Department of Mechanics, Thákurova 7, \\ 16629 Prague 6, Czech Republic \\ * corresponding author: jiri.nemecek.1@fsv.cvut.cz (Ph.D. candidate)
}

\begin{abstract}
This study presents a numerical simulation of the mini-slump flow test performed on the Haegerman table, according to EN 1015-3. The two-dimensional axisymmetrical Volume of fluid (VOF) model is used for the calculation. Various scenarios with the different model set up and boundary conditions were calculated to show how the results are affected. Simulations with different lifting velocities were carried out and compared to the case with instantaneous demolding, which is commonly used in numerical simulations. Also, the effect on results is shown between noslip and specific shear boundary conditions on the mold. The results of simulations indicate that mold lifting should be considered in numerical modeling even if calculation time is highly prolonged. Lifting velocities should not exceed the value of $0.2 \mathrm{~m} / \mathrm{s}$ in order to maintain laminar flow and stable calculation.
\end{abstract}

KEywords: Cement paste, CFD modeling, rheology, slump test, volume of fluid.

\section{INTRODUCTION}

Concrete is a mixture of cement, fine, coarse aggregate, admixtures, and water. After mixing initial components with water, a fluid suitable for filling formwork is created. Each mixture has different rheological properties depending on its composition, which could be characterized by various experimental tests such as slump test, L-box, V-funnel tests, viscosimeter, and vibration testing [1]. The most commonly used is the cone slump testing for its feasibility and reproducibility on in-situ and ex-situ conditions. The test is conducted with a metallic cone opened at both ends, which is placed on a metallic/wooden/glass table. The fresh mixture is filled inside the cone, and then the metallic cone is lifted in a vertical direction. When the initial shear stress of the mixture is overcome, the concrete flows by gravity $[2]$.

The test is commonly simulated by numerical modeling using computational fluid dynamics (CFD) approach. Most of the studies modeled the slump-test with instantaneous demolding [2-4]. However, such a boundary condition (b.c.) is leading to a higher slump spread. This behavior is then artificially correlated by changing constants of the material model. Gao investigated the effect of a cylindrical mold lifting on the slump test of kaoline clay [5]. The results suggest the mold lifting velocity has a significant influence on the final slump spread.

In this study, the setting up of a numerical model of the mini-cone slump test is shown. The effect of different boundary conditions on the final slump spread

\footnotetext{
${ }^{1} \mathrm{Ph}$. D. candidate at CTU in Prague

${ }^{2}$ Associate Professor at CTU in Prague
}

is investigated.

\section{NumERICAL SIMULATION}

\subsection{VOLUME OF FLUID MODEL}

The Eulerian multiphase model is the most complex model which can calculate dispersed-continuous phase interaction and continuous-continuous phase interaction. Typically dispersed micrometer or millimeter size particles could be solid particles, liquids droplets, or bubbles of gas which are dissolved in a continuous phase. Continuous-continuous phase interaction is represented by two immiscible phases, e.g., concrete and air. The Eulerian model is solving continuity and momentum equations individually for each phase. The simplified version of the Eulerian model used for continuous-continuous phase interaction is the Volume of Fluid (VOF) model. The VOF model is solving only one continuity equation and one momentum equation and tracks each phase's volume fraction $[6,7]$. The continuity equation could be simplified as

$$
\nabla \cdot \vec{v}=0
$$

where where $\vec{v}$ is the velocity and time field. A single momentum equation is written as

$$
\begin{aligned}
& \frac{\partial}{\partial t}(\rho \vec{v})+\nabla \cdot(\rho \vec{v} \vec{v})=-\nabla p+\nabla \cdot\left[\eta\left(\nabla \vec{v}+\nabla \vec{v}^{T}\right)\right] \\
& +\rho \vec{g}+\vec{F},
\end{aligned}
$$

where $p$ is the static pressure, $g$ is the gravitational acceleration, $\vec{F}$ is the force resulting from surface tension at the interface, and $\rho$ is the fluid density and $\eta$ is the apparent viscosity. 

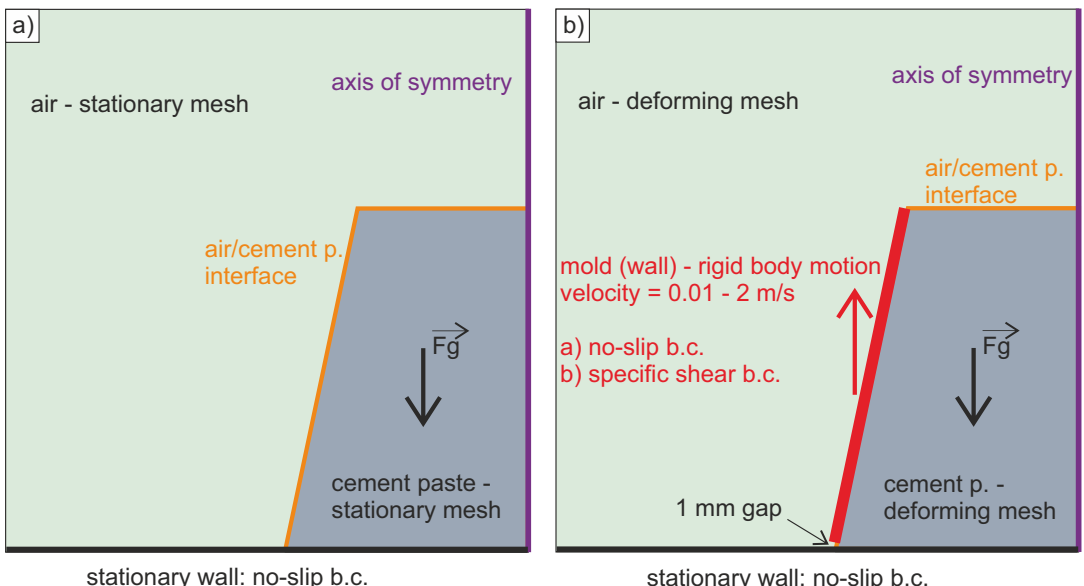

FiguRE 1. Schematic set up of mini-slump (symmetric part of the cone is shown) test with shown boundary conditions a) without mold b) with mold.

In VOF model volume fraction of phase in an individual cell is set to either 0 (none phase is present) or 1 (phase is fully present). In the sharp interface region, the value in the cell could range from 0 to 1. In order to preserve a sharp interface, one of few discretization schemes has to be used. The more details about discretization schemes could be found in ANSYS Fluent theory guide [6]. In the current study, explicit scheme formulation with a geometric reconstruction scheme [8] was used to solve the volume fraction equation.

\subsection{MATERIAL MODEL}

Computationally, the concrete can be assumed as non-Newtonian fluid. Several models, such as Bingham or Herschel-Bulkley, allow to model concrete flow. In both models it is required to overcome the Yield stress threshold to initiate flow, the flow of fluid is further prescribed with plastic viscosity. For the Bingham plastic model, after the stress is initiated, the shear stress is a linear function of the shear rate, and fluid behaves as Newtonian. Herchel-Bulkley's model exhibits shear-thinning behavior. In ANSYS Fluent for shear rate $\dot{\gamma}>$ critical shear rate $\dot{\gamma}_{c}$ the viscosity is calculated as

$$
\eta=\frac{\tau_{0}}{\dot{\gamma}}+k \dot{\gamma}^{n-1}
$$

where $\tau_{0}$ is the Yield stress threshold, $k$ is the consistency index, and $n$ is the power-law index. For Bingham model $n=1$, for Herchel-Bulkley: $0<n<1$. Critical shear rate $\dot{\gamma}_{c}$ is the point beyond which material acts like very viscous fluid [6]. For $\dot{\gamma}<\dot{\gamma}_{c}$ the viscosity is expressed by equation

$$
\eta=\frac{\tau_{0}\left(2-\frac{\dot{\gamma}}{\dot{\gamma}_{c}}\right)}{\dot{\gamma}_{c}}+k \dot{\gamma}_{c}^{n-1}\left[(2-n)+(n-1) \frac{\dot{\gamma}}{\dot{\gamma}_{c}}\right]
$$

\section{NumERICAL MODELING}

The presented numerical model was used to reproduce the slump flow test of mini-cone on the Haegerman table described in EN 1015-3. The dimensions of the mini-cone are defined as following: upper radius $=70 \mathrm{~mm}$, bottom radius $=100 \mathrm{~mm}$, height $=60 \mathrm{~mm}$. The numerical model was solved as a $2 \mathrm{D}$ axisymmetric task. In general, two approaches could be followed to obtain slump spread and height of the mini-cone. In the first approach, the mold is not taken into account in the simulation. The mesh is stationary, and the overall calculation time is short. In the second approach, the mold and its lifting are added to the simulation, which requires dynamically changing mesh. The calculation time is then significantly prolonged. One set of parameters of the Herschel-Bulkley material model was used in the modeling to show differences in boundary conditions. The set of parameters was chosen for typical cement paste without admixtures as follow: $\tau_{0}=25 \mathrm{~Pa}, k=$ $10 \mathrm{~Pa} \cdot s^{n}, n=0.5$, density $\rho=1800 \mathrm{~kg} / \mathrm{m}^{3}$. Critical shear stress was also fixed at a value $\dot{\gamma}_{c}=0.005 \mathrm{~s}^{-1}$. The air was modeled as Newtonian fluid with density $=1.225 \mathrm{~kg} / \mathrm{m}^{3}$ and viscosity $=1.7894 \mathrm{e}-05 \mathrm{~kg} / \mathrm{m} \cdot \mathrm{s}$.

\subsection{Slump TeSt Without MOLD}

The schematic situation of the model without mold is shown in Fig. 1a. The domain of $300 \times 100 \mathrm{~mm}$ was filled with linear quadrilateral cells. The frequency of cells near the base wall was increased to capture the velocity profile correctly, see Fig. 2a. No-slip boundary condition was prescribed on the base wall. The initial trapezoidal shape of cement paste acts in the gravitational field, which is the only external force acting on the mini-slump.

\subsection{Slump TeSt With MOLD LifTiNG}

The scheme of the model with mold is shown in Fig. 1b. The mold with thickness of $8 \mathrm{~mm}$ was added on the left side mini-cone, and its lifting was modeled 

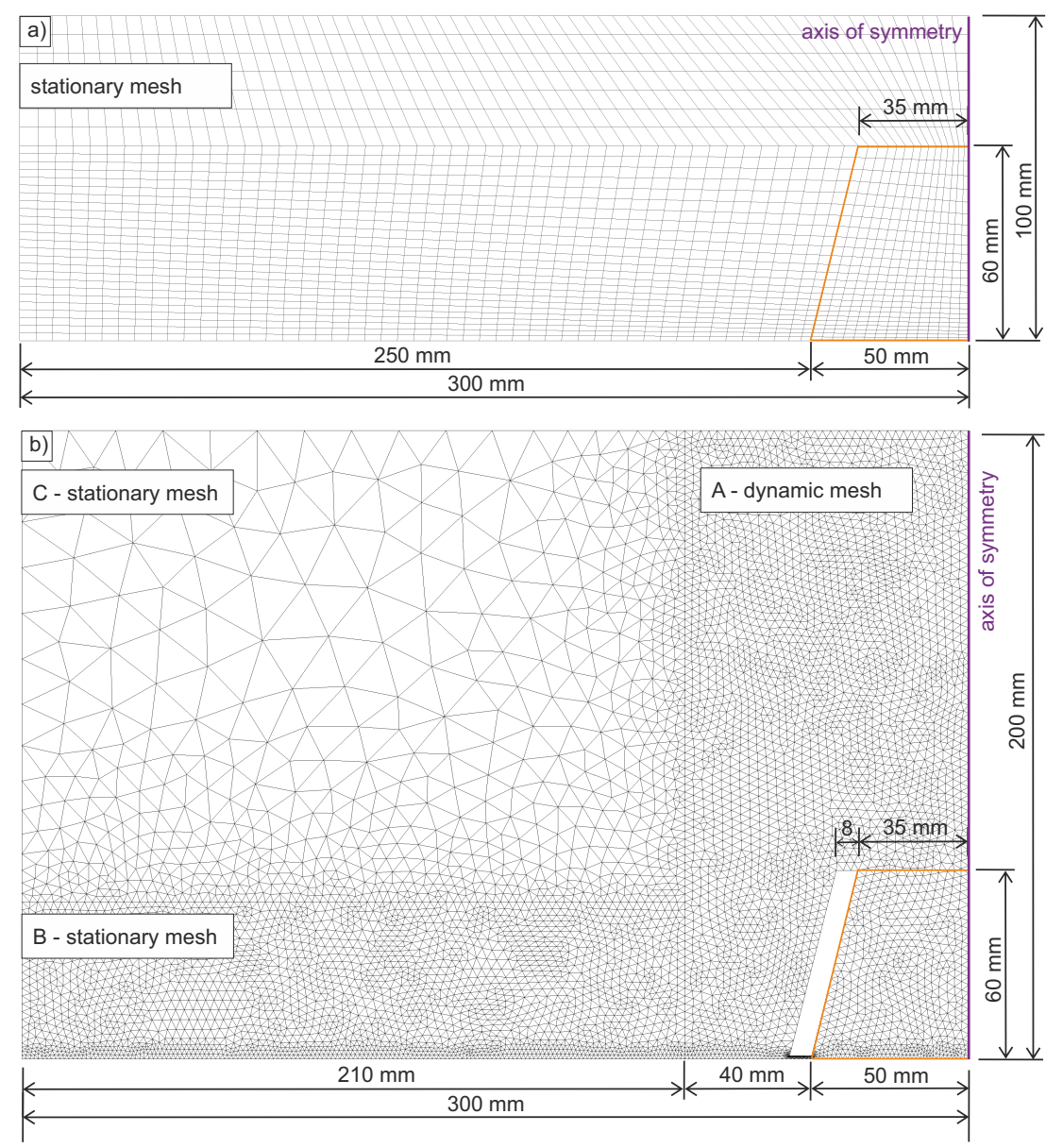

Figure 2. Mesh used for the slump test modeling a) without mold b) with mold.

as rigid body motion in the vertical direction. Several cases with various velocities: $0.01 \mathrm{~m} / \mathrm{s} ; 0.05 \mathrm{~m} / \mathrm{s}$; $0.2 \mathrm{~m} / \mathrm{s} ; 2.0 \mathrm{~m} / \mathrm{s}$ were calculated to show differences in final slump spread. Such a problem requires a dynamically changing mesh of the domain. Triangular cells were chosen as most suitable for the analysis. In order to save calculation time, only one part of the domain was used for adaptive meshing. Thus, the domain $300 \times 200 \mathrm{~mm}$ was then divided into three regions. The first part $(\mathrm{A})$ around the mold and cement domain was filled with the small size, $2.5 \mathrm{~mm}$ triangular cells. In this part, adaptive changes in the mesh: smoothing and remeshing were applied. The second part (B) is above the rest of the table with $2.5 \mathrm{~mm}$ cells. The cells were smoothened only above the table (wall b.c.). The third part (C) was filled with larger $15 \mathrm{~mm}$ cells. Both the second and third parts of the mesh stayed stationary. The mesh is shown in Fig. 2b. In the case of adaptive meshing in ANSYS Fluent, the divergence problems occurred on the interface of mold and table (wall-wall boundary condition) where, after the initial lifting, the mesh is missing under the mold. To overcome such a difficulty $1 \mathrm{~mm}$ of mold was cut off in model, which should have minimal effect on the results. The gap was filled with triangular cells and air particles. The mold wallcement interface was modeled with two variants: a) no-slip b.c., b) specific shear b.c. with a value of $0.3 \mathrm{~Pa}$.

\section{Results AND Discussion}

Fig. 3 shows three variants of the slump spreading results displayed in five different times. The first variant is the slump spread without mold (Fig. 3a). After the calculation begins, all the volume of cement paste starts to flow. No obstacle prevents cement from movement, and due to the high inertia effect, the slump spread reaches the highest value $(2 \times 99.8=199.6 \mathrm{~mm})$. The result with considering mold lifting with a velocity of $0.05 \mathrm{~m} / \mathrm{s}$ and no-slip b.c. prescribed on the mold and table is shown in Fig. 3b. Obviously, the mold is partly holding the whole cement volume from motion, which is the factor decreasing the final spread $(2 \times 87.3=174.6 \mathrm{~mm})$ which is $12.5 \%$ decrease in slump spread $(199.6 \mathrm{~mm})$ beside no-mold condition. Due to no-slip b.c., part of the cement paste is sticked to the mold, which is another factor reducing the final spread. The third case (Fig. 3c) shows mold lifting $(v=0.05 \mathrm{~m} / \mathrm{s})$ with shear b.c. prescribed on the mold, the chosen value $0.3 \mathrm{~Pa}$ represent almost no shear between mold-cement interface. In this case, the cement is not sticking to the mold, which functions only as an obstacle. The overall result of slump spread $(2 \times 92.7=185.4 \mathrm{~mm})$ is 
a) no-mold
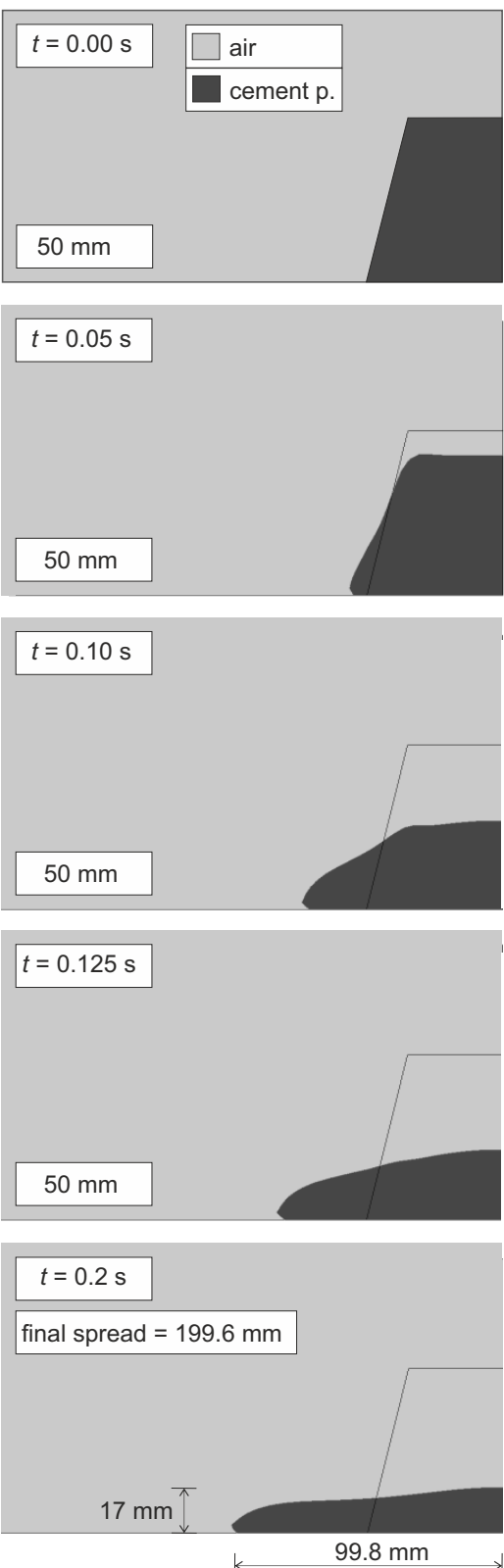

b) mold lifting $=0.05 \mathrm{~m} / \mathrm{s}$; no-slip b.c.
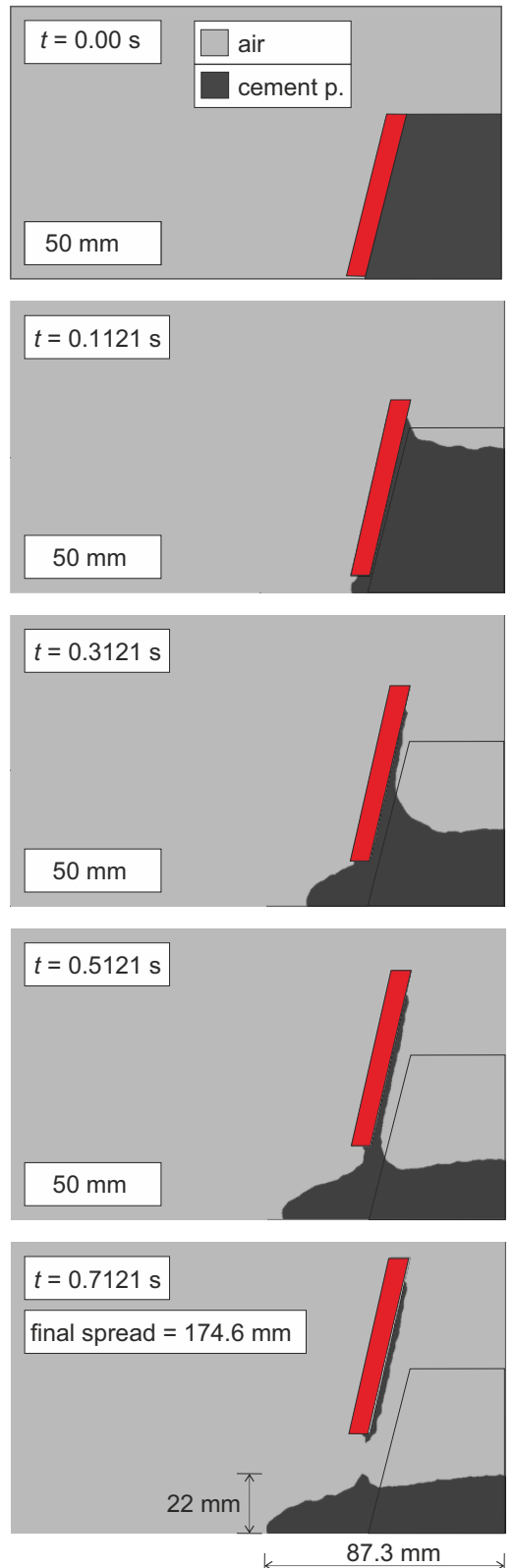

c) mold lifting $=0.05 \mathrm{~m} / \mathrm{s}$; specific shear b.c.
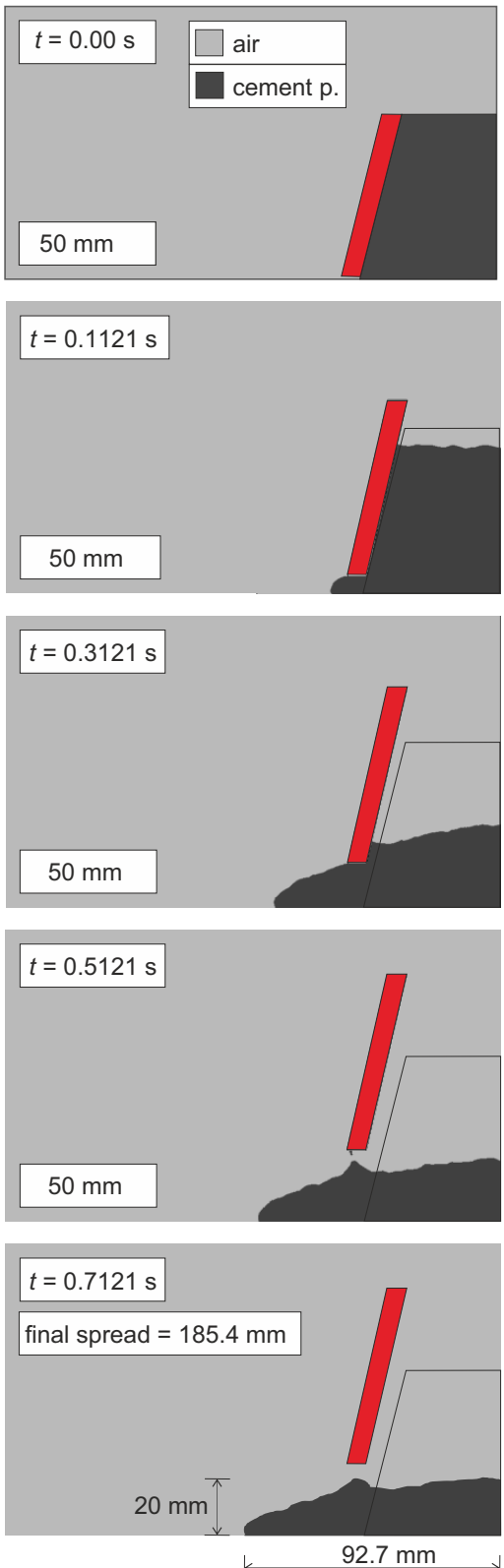

FiguRE 3. Results of calculation of mini-slump test in five different times: a) without mold b) with mold lifting $(v=0.05 \mathrm{~m} / \mathrm{s})$ and no-slip b.c. $\mathrm{c})$ with mold lifting $(v=0.05 \mathrm{~m} / \mathrm{s})$ and specific shear b.c. on the mold $(0.3 \mathrm{~Pa})$.

higher than in the previous case with no-slip b.c. but lower than in model without the mold. The decrease in the slump spread is only $7.1 \%$ besides the no-mold case. In terms of reproducing the real behavior of the mini-slump test, the case in Fig. 3b is most promising. Since during the experiment, cement paste is always sticking to the steel mold. Applying the real value of friction would be even more suitable. However, establishing the coefficient's value on a water wet steel-cement paste interface is not a simple task since the amount of water used, and admixtures used differs in each individual experiment.

The results of the final spread and slump are summarized in Tab. 1 and shown in Fig. 4. It is apparent that lifting velocity affects overall results. Basi-

\begin{tabular}{cccc}
\hline & $\begin{array}{c}\text { Spread } \\
{[\mathrm{mm}]}\end{array}$ & $\begin{array}{c}\text { Slump } \\
{[\mathrm{mm}]}\end{array}$ & $\begin{array}{c}\text { Increase of } \\
\text { spread }\end{array}$ \\
\hline$v=0.01 \mathrm{~m} / \mathrm{s}$ & 171.8 & 23.4 & $+0.0 \%$ \\
$v=0.05 \mathrm{~m} / \mathrm{s}$ & 174.6 & 22.1 & $+1.6 \%$ \\
$v=0.20 \mathrm{~m} / \mathrm{s}$ & 176.4 & 22.2 & $+2.7 \%$ \\
$v=2.00 \mathrm{~m} / \mathrm{s}$ & - & - & - \\
no-mold & 199.6 & 17.2 & $+16.2 \%$ \\
\hline
\end{tabular}

TABLE 1. Results of spread and slump calculated with different mold lifting velocities and no-slip b.c.

cally, for lower lifting velocity $(v=0.01-0.20 \mathrm{~m} / \mathrm{s})$, the spread is increased when the lifting velocity is in- 


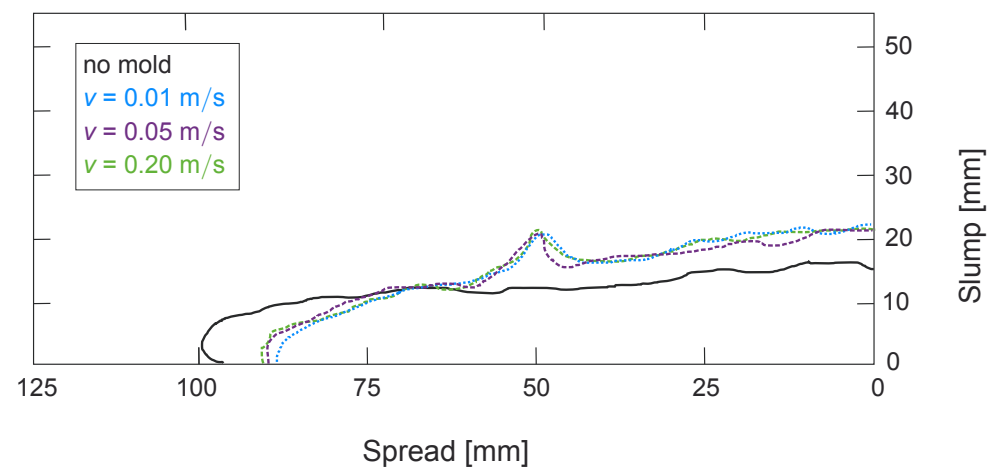

FigURE 4. Results of spread and slump calculated with different mold lifting velocities.

creased. However, the difference between the smallest $(v=0.01 \mathrm{~m} / \mathrm{s})$ and maximum $(v=0.20 \mathrm{~m} / \mathrm{s})$ spread is only $2.7 \%$. Which is much lower than the difference $16.3 \%$ between mold and no-mold lifting. The high lifting velocities $2 \mathrm{~m} / \mathrm{s}$ bring the cement paste to motion where other forces than gravitational are acting. The slumping process is not smooth, and the results are most probably incorrect due to the turbulent flow and calculation instability. Similar findings were found in Gao's study, where different geometry and material were used [5].

The calculation time necessary for the model without a mold and stationary mesh is about 5 minutes. The time needed for calculation with dynamic mesh and mold lifting is 3-7 hours depending on the b.c. The total calculation time could be reduced using variable time step where the smaller time step is necessary at the beginning of the calculation and could be further increased during the calculation.

\section{Conclusions}

The present work provides a general study of setting up a numerical model of slump flow test and shows how different boundary conditions could affect the results. The calculation was done on typical cement paste using only one set of parameters of the HerchelBulkley model to neglect different mixtures' effect. Several conclusions are made.

- The consideration of the mold lifting during calculation has a significant effect on results. The final slump spread is increased by $12-16 \%$ when the mold lifting is not used in the calculation.

- Mold lifting velocity has only a small effect on overall slump spread while slow lifting velocities are used. Slow lifting velocities are considered in the range of $0.01-0.20 \mathrm{~m} / \mathrm{s}$, which should be typical lifting velocities used in laboratory conditions. The difference in slump spread between minimum and maximum velocity was calculated as $2.7 \%$.

- Lifting velocities with higher than $2 \mathrm{~m} / \mathrm{s}$ bring fluid to turbulent motion, which is not recommended to calculate in ANSYS Fluent with the HerchelBulkley model suitable for laminar flow. Moreover, these velocities are not commonly used in the experiments.

- Consideration of mold lifting and dynamic remeshing in the calculation results in increasing calculation time from minutes to hours.

\section{ACKNOWLEDGEMENTS}

Financial support of the Technological Agency of the Czech Republic (Trend FW-01010521) and the Grant Agency of the Czech Technical University in Prague (SGS20/107/OHK1/2T/11) are gratefully acknowledged.

\section{REFERENCES}

[1] C. Ferraris, F. De Larrard, N. Martys. Fresh concrete rheology: recent developments. Materials Science of Concrete VI, Amer Cer Soc Ed S Mindess, J Skalny pp. 215-241, 2001.

[2] A. Bouvet, E. Ghorbel, R. Bennacer. The mini-conical slump flow test: Analysis and numerical study. Cement and Concrete Research 40(10):1517 - 1523, 2010. DOI:https://doi.org/10.1016/j.cemconres.2010.06.005.

[3] F. Dufour, G. Pijaudier-Cabot. Numerical modelling of concrete flow: homogeneous approach. International Journal for Numerical and Analytical Methods in Geomechanics 29(4):395-416, 2005. https:// onlinelibrary.wiley.com/doi/pdf/10.1002/nag.419 DOI:10.1002/nag.419.

[4] N. Roussel, P. Coussot. "fifty-cent rheometer" for yield stress measurements: From slump to spreading flow. Journal of Rheology 49, 2005. DOI:10.1122/1.1879041.

[5] J. Gao, A. Fourie. Spread is better: An investigation of the mini-slump test. Minerals Engineering 71:120 $132,2015$.

DOI:https://doi.org/10.1016/j.mineng.2014.11.001.

[6] I. Ansys. Ansys fluent theory guide. vol 15317:514, 2011.

[7] N. D. Katopodes. Chapter 12 - volume of fluid method. In N. D. Katopodes (ed.), Free-Surface Flow, pp. 766 - 802. Butterworth-Heinemann, 2019. DOI:https://doi.org/10.1016/B978-0-12-8154854.00018-8.

[8] D. Youngs. Time-Dependent Multi-material Flow with Large Fluid Distortion, vol. 24, pp. 273-285. 1982. 